\title{
La evaluación en los procesos de aprendizaje del diseño arquitectónico
}

Antonio Rosales Hernández / Humberto Vázquez Ramírez

L

a enseñanza de la arquitectura en la Universidad Autónoma de Aguascalientes (UAA) en las primeras generaciones no contaba con una metodología para el diseño y los sistemas de evaluación, pues éstos eran determinados por cada profesor o grupo de profesores de acuerdo a su percepción e interpretación de la asignatura. A partir de la primera revisión curricular se implementó un solo proceso de diseño, la metodología para el diseño arquitectónico (Andrade Muñoz y colaboradores, 1988), que dio origen a la asignatura del mismo nombre y fue aplicada en todos los talleres de diseño arquitectónico a partir de la segunda revisión curricular. Esta acción sin duda fue un camino hacia la objetividad en los procesos subjetivos de la creatividad y un logro de la carrera de Arquitectura de la UAA, a nivel nacional. Con esto se volvió posible evaluar un proceso de diseño y retroalimentar al estudiante, a partir del manejo de un solo lenguaje; fue viable, además, evaluar un proceso de diseño de una manera más argumentada o racional; sin embargo, la evaluación de la creatividad y sus procesos de pensamiento siguen teniendo un sesgo subjetivo; al respecto, Edward T. White menciona lo siguiente: "por su naturaleza creativa, por ese proceso del pensamiento que no se ha podido racionalizar [...] la Arquitectura es una actividad científica pero también artística" (White, 1973: 25).

En este sentido, el Modelo Educativo Institucional (MEI) de la UAA promueve una educación centrada en el alumno, que pretende vincular la teoría y la práctica en un aprendizaje basado en la solución de problemas, con el objeto de que el alumno mejore su autoestima y el trabajo colaborativo de una forma libre y responsable. Por ello, la evaluación de los aprendizajes es una función que compete tanto a los profesores como a los estudiantes, y que se da en distintos momentos o etapas del proceso educativo: evaluación diagnóstica, evaluación procesual y evaluación sumativa. Asimismo, es necesario que se evalúen los aprendizajes de una manera integral, es decir, considerando no sólo los conocimientos, sino también las habilidades y las actitudes hacia el aprendizaje, que se contemplan curricularmente y en los objetivos y unidades de aprendizaje.

\section{Los sistemas e instrumentos de evaluación}

Existen algunos aspectos que habría que considerar en los sistemas y diseño de instrumentos de evaluación, específicamente en los talleres de diseño arquitectónico:

- El objeto de la evaluación (para qué evaluamos).

- El sujeto de la evaluación (qué evaluamos, un proceso o un producto).

- La implementación de la evaluación (cómo evaluamos, cómo hacer objetiva la subjetividad de la ponderación, entre otros).

El objeto de la evaluación (para qué evaluamos). ¿Cuál es el objeto de la evaluación? ¿Es medir en el alumno el procedimiento o proceso para obtener un conocimiento o medir en el alumno el nivel de aplicación de un conocimiento que adquiere? El objeto de la evaluación es medir en el estudiante el proceso de aprendizaje de un conocimiento experimentado en un taller de diseño, ya que el conocimiento se construye a partir de la experimentación. 
Se debe tener muy claras las referencias del nivel de conocimiento y aprendizaje que se va a cubrir por el estudiante en cada asignatura y en cada semestre para poder medir de una manera objetiva. Asimismo, académica e institucionalmente, estas referencias están determinadas curricularmente, y aparecen claramente explícitas en los programas de las materias que los grupos colegiados como las academias revisan periódicamente, en particular a nivel de cumplimiento de objetivos (generales y particulares) de una asignatura determinada.

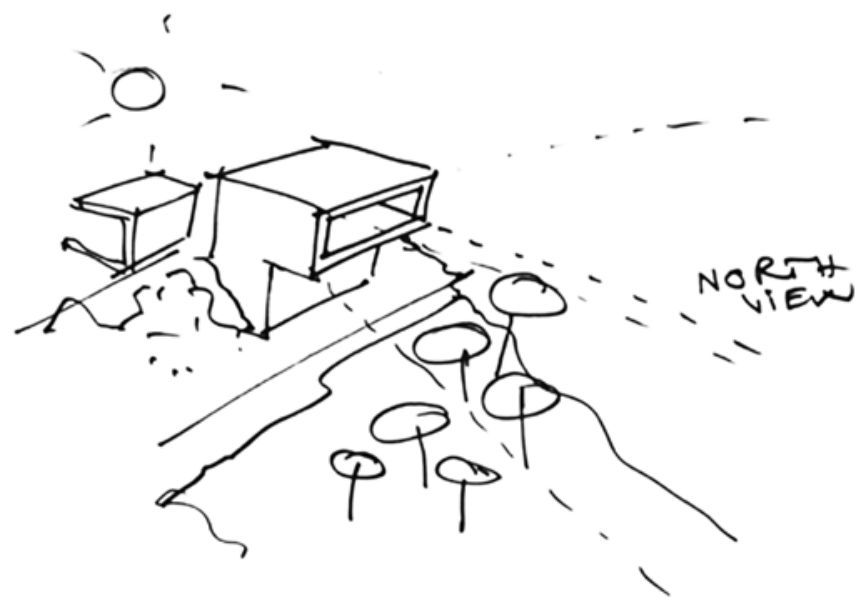

Foto 2. Croquis del estudio de contexto. Fuente: extraída de internet.

Por lo tanto, el objeto de la evaluación es medir en el alumno el proceso de aprendizaje de un conocimiento experimentado, es decir, el nivel del cumplimiento de los objetivos de la asignatura de taller de diseño.

El sujeto de la evaluación. ¿Qué es lo que evaluamos, el aprendizaje del alumno como resultado de un proceso, o el producto de un proceso de enseñanza y aprendizaje? En los talleres de diseño se evalúa el proceso de aprendizaje y el producto que resulta de éste. En dicho proceso "interviene más de una variable que la sola aplicación de un procedimiento de enseñanza-aprendizaje y son éstas las que permiten que el conocimiento se vaya construyendo" (Soto Martínez, 2012: s/p).

Fundamentalmente, las variables que intervienen en la construcción del conocimiento son las habilidades y actitudes que demuestra el alumno ante el reto del aprendizaje; estas referencias están determinadas explícitamente en los currículos por carrera. Por ejemplo, el egresado de la carrera de Arquitectura (Plan de estudios, 2001) contará con conocimientos, habilidades y actitudes

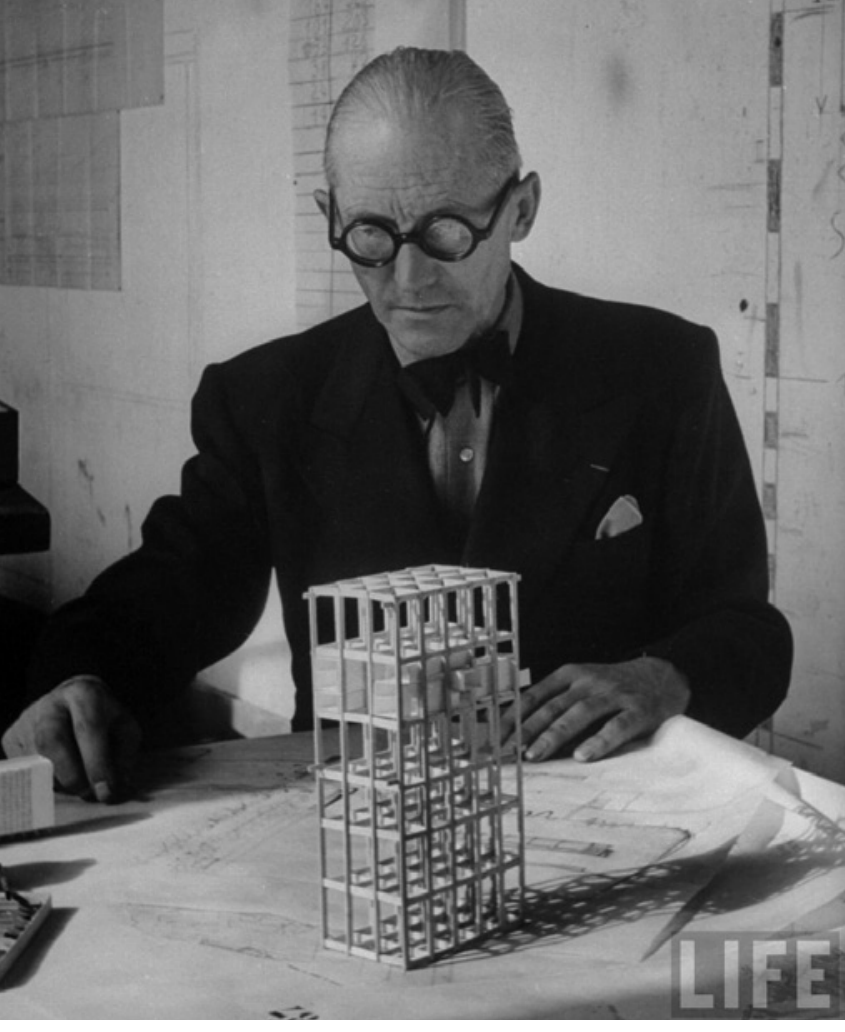

Foto 1. Arquitectura es cosa de arte, un fenómeno de emociones, que queda fuera y más allá de las cuestiones constructivas. Le Corbusier. http://www.catalogoarquitectura.com/ Blog/post/2012/02/15/Alemania-1910-Arquitectura-y-coincidencias.aspx. Noviembre 2012.

particulares que pueden definirse específicamente por área, por semestre, o incluso por asignatura, en el programa de la materia y en el instrumento de evaluación.

La implementación de la evaluación (¿cómo evaluamos?; la subjetividad de la ponderación). Por su naturaleza misma, la creatividad está influida por otros aspectos, uno de ellos tiene que ver con lo que menciona Molina Ayala en el siguiente párrafo: "la manera de ver la vida, e interpretar lo cotidiano; el arquitecto diseña en función de su interpretación y postura personal de pensamiento, sin embargo, hay que considerar la forma de pensar del usuario o habitador y su contexto" (Molina Ayala, 2012: s/p).

Otro aspecto que influye el proceso creativo del diseño arquitectónico según Acevedo Salomao es el hecho de que "responde a la cultura, el lenguaje, la tecnología, las interacciones sociales y medioambientales de un individuo y su grupo social, pues ésta genera patrones" (Acevedo Salomao, E., 2012: s/p).

\footnotetext{
Como el caso de las definidas en el artículo "La evaluación en los procesos del diseño arquitectónico. Una experiencia docente", de Humberto Vázquez, Rafael Vázquez y Patricia Villalpando (2011).
} 


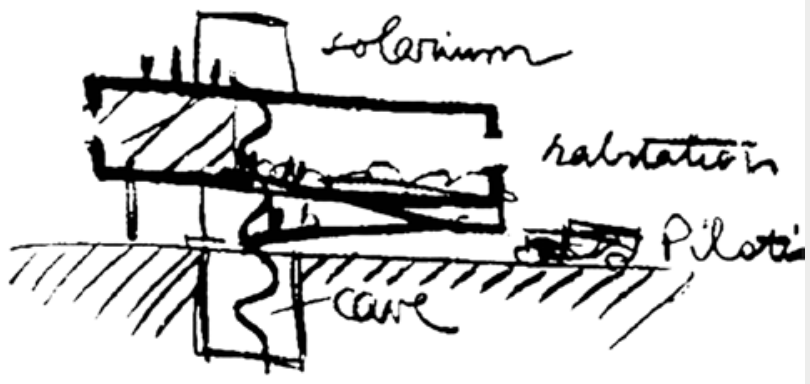

Foto 4. Le Corbusier. Concepto. Fuente: extraída de internet.

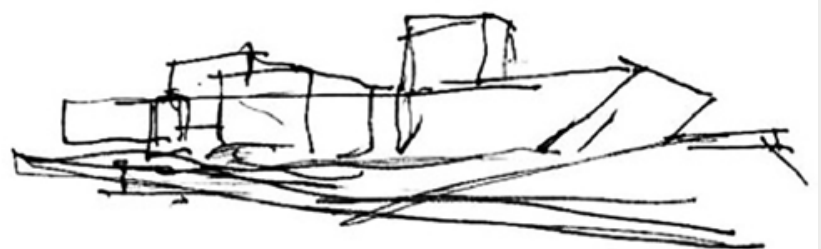

Foto 5. Teodoro González de León. Concepto. Fuente:

http://www.jornada.unam.mx/2a2/03/21/cultura/a03n1cul

8. Es conveniente el uso de un instrumento de evaluación que considere los acuerdos respecto a los ítems que se van a evaluar por parte de los profesores.

\section{Fuentes de consulta}

Acevedo Salomao, E. (2012). Reflexiones sobre el espacio habitado y formas de vida. 87 Reunión de ASINEA, mayo de 2012, CCDC, UAA.

Andrade Muñoz M., Sánchez de Aguilera M. E., Sotomayor de la Serna M. G., (1988). El método de diseño. Un método científico. México: UAA.

Canseco, H. y Galaviz Rebollozo, D. (2012). La importancia de la enseñanza-aprendizaje para la formación de arquitectos conscientes y responsables. $87^{\text {a }}$ Reunión de ASINEA, mayo de 2012, CCDC, UAA.

Molina Ayala, M.E. (2012). El diseño del espacio antrópico como producto de la cosmovisión y de la abstracción. $87^{\text {a }}$ Reunión de ASINEA, mayo de 2012, CCDC, UAA.

Plan de Estudios de la Licenciatura en Arquitectura 2001. Departamento de Diseño del Hábitat, CCDC, UAA.

Plan de Estudios de la Licenciatura en Arquitectura 2012 (2012). Departamento de Diseño del Hábitat, CCDC, UAA.

Soto Martínez, J. (2012). Foro de Innovación Educativa 2012. 27 de junio de 2012, CCDC, UAA.
Universidad Autónoma de Aguascalientes. (2007). Modelo Educativo Institucional. Correo Universitario, sexta época, núm.16, publicado el 15 de marzo de 2007. México: UAA.

Vázquez, H., Vázquez, R. y Villalpando, P. (2011). La evaluación en los procesos del diseño arquitectónico. Una experiencia docente. Docere. Revista del Departamento de Formación y Acualización Académica, No. 4 , junio.

White, E.T. (1973). Sistemas de ordenamiento: una introducción al diseño arquitectónico. México: Ed. Trillas.

White, E.T. (1975). Manual de conceptos y formas arquitectónicas. México: Ed. Trillas. 